\title{
Immunotherapy in small cell lung cancer: one step at a time: a narrative review
}

\author{
Daphne W. Dumoulin ${ }^{1}$, Anne-Marie C. Dingemans ${ }^{1,2}$, Joachim G. J. V. Aerts ${ }^{1}$, Jordi Remon ${ }^{3}$, \\ Dirk K. M. De Ruysscher ${ }^{4}$, Lizza E. L. Hendriks ${ }^{2}$
}

${ }^{1}$ Department of Pulmonary Medicine, Erasmus MC Cancer Institute, Rotterdam, The Netherlands; ${ }^{2}$ Department of Respiratory Medicine, Maastricht University Medical Centre, GROW School for Oncology and Developmental Biology, Maastricht, The Netherlands; ${ }^{3}$ Department of Medical Oncology, Centro Integral Oncológico Clara Campal Barcelona (CIOCCB), Hospital HM Delfos, HM Hospitales, Barcelona, Spain; ${ }^{4}$ Department of Radiation Oncology (MAASTRO Clinic), Maastricht University Medical Centre, GROW School for Oncology and Developmental Biology, Maastricht, The Netherlands

Contributions: (I) Conception and design: DW Dumoulin, AMC Dingemans, LEL Hendriks; (II) Administrative support: DW Dumoulin, LEL Hendriks; (III) Provision of study materials or patients: DW Dumoulin, LEL Hendriks; (IV) Collection and assembly of data: DW Dumoulin, LEL Hendriks, AMC Dingemans; (V) Data analysis and interpretation: All authors; (VI) Manuscript writing: All authors; (VII) Final approval of manuscript: All authors.

Correspondence to: Daphne W. Dumoulin, MD. Department of pulmonary diseases Erasmus MC, Erasmus MC Cancer Institute, Rotterdam, The Netherlands. Email: d.dumoulin@erasmusmc.nl.

\begin{abstract}
Chemotherapy with or without radiotherapy has been the standard of care for many years for patients with small cell lung cancer (SCLC). Despite exceptionally high responses (up to 80\%) with chemotherapy, the majority of patients relapse rapidly within weeks to months after treatment completion. Therefore, new and better treatment options are necessary. Recently, synergistic activity has been reported for the addition of immune checkpoint inhibitors (ICI) to standard platinum-based chemotherapy in the therapeutic strategy of advanced SCLC. For the first time after several decades, a significant survival improvement was achieved for this population. However, the overwhelming majority of patients do not respond to ICI, or relapse rapidly. There is need for better knowledge about the biology, histopathologic features, and molecular pathways of SCLC. This can probably help to identify the optimal predictive biomarkers, which are warranted to develop an individual therapeutic strategy including the rational use of a combination of immunotherapeutic agents. Here, we provide an overview of the rationale for and clinical results of the completed and ongoing trials using different strategies of immunotherapy in SCLC. In addition, opportunities for further improvement of therapies will be discussed, including the addition of radiotherapy, co-stimulatory antibodies, and other immune modifying agents.
\end{abstract}

Keywords: Small cell lung cancer (SCLC); immunotherapy; checkpoint inhibition

Submitted May 03, 2020. Accepted for publication Oct 21, 2020.

doi: $10.21037 /$ tlcr-20-630

View this article at: http://dx.doi.org/10.21037/tlcr-20-630

\section{Introduction}

Lung cancer is a major global health concern, and causes 1.6 million deaths yearly (1). Small cell lung cancer (SCLC) represents about $10-15 \%$ of all lung cancers in Western countries. Smoking is the single most risk factor for developing SCLC (2). With an incidence of 1-5/10,000, SCLC is recognized as an orphan disease (3). At diagnosis, approximately $70 \%$ of patients already have detectable distant metastases (4). The prognosis remains poor with a 5 -year overall survival (OS) of $25-33 \%$ for limited disease SCLC (LD-SCLC) and $3 \%$ for extensive disease (EDSCLC) (5-7). Unfortunately, little progress has been made over the last decades (8).

For many years, chemotherapy has been the backbone 
of the treatment for all stages. Despite exceptionally high responses with chemotherapeutical agents like etoposide plus platinum, the majority of patients relapse rapidly (9). In second line, the only approved treatment is topotecan, with significant toxicity, poor response rates of $24 \%$ and a median OS of 6 months (10). Immune checkpoint inhibitors (ICI) such as programmed cell death 1 (PD-1) inhibitors or the programmed cell death-ligand1 (PD-L1) inhibitors have revolutionized the treatment of stage III and IV non-small cell lung cancer (NSCLC), reporting survival improvement either as monotherapy or in combination with chemotherapy (11-15), but their role in SCLC is less established. This narrative review provides an overview of the rationale for, and clinical results of immunotherapy in SCLC. Furthermore, ongoing challenges and directions for research are discussed.

We present the following article in accordance with the Narrative Review reporting checklist (available at http:// dx.doi.org/10.21037/tlcr-20-630).

\section{Methods}

For this narrative review, a search of the literature on PubMed (last search date Oct 4, 2020), as well as the meeting libraries of the largest oncological conferences (World Conference on Lung Cancer, ASCO, ESMO) was performed (last search date Oct 4, 2020). Only abstracts of full publications in English were considered eligible.

\section{Rationale for immunotherapy in SCLC}

Tumors can escape immune surveillance by a number of mechanisms. Inhibitory checkpoints have been recognized to play a key role in this process. By blocking the PD-1 receptor on $\mathrm{T}$ cells or PD-L1 receptor on tumor cells, cytotoxic antitumor activity by exhausted CD8+ T cells can be restored (16). Furthermore, the co-inhibitory receptor cytotoxic T lymphocyte associated antigen 4 (CTLA-4) that is expressed on T cells, can bind to CD80 and CD86 on antigen presenting cells with a higher affinity than the costimulatory receptor CD28, thereby blocking activation and proliferation of T cells (17).

Theoretically, SCLC should be an immunogenic tumor type due to several characteristics. Long-term exposure to carcinogens in cigarettes induces high rates of somatic mutations (18). This is reflected in the high tumor mutational burden (TMB) of SCLC compared with other tumors (19). Tumors with a high TMB are generally presenting more neoantigens, which can induce an immune response. In different tumor types, high TMB was indeed associated with response to ICI, although rarely, some cancers with low TMB are amongst the best responders to ICI $(20,21)$. In addition, SCLC is frequently associated with paraneoplastic syndromes such as LambertEaton myasthenic syndrome (LEMS) and anti-Hu $(22,23)$. Paraneoplastic syndromes occur as a result from an immune response targeting antigens expressed by both SCLC and healthy tissues, in this example the central nervous system. Patients with neurological paraneoplastic syndromes may have a more inflamed tumor micro-environment and better OS compared to those without (24), and it has been suggested that the occurrence of baseline neuronal antibodies may be a potential predictive marker for the efficacy of ICI in SCLC $(25,26)$.

In contrast to NSCLC, PD-L1 expression [associated with anti-PD-(L)1 efficacy] is lower in SCLC. Previous cohorts have reported PD-L1 tumor cell positivity ranging from $10-19 \%$, with less than $5 \%$ of tumors express PDL1 $\geq 50 \%$ (27), although more recent data report PD-L1 expression in up to $39 \%$ of cases (28). This may be related to the finding that levels of inflammatory cells, such as tumor infiltrating lymphocytes (TILs) are reduced in SCLC compared with NSCLC (29). These cells are essential for an effective anti-tumor response. In addition, the expression of major histocompatibility antigens (MHC) class I and II are also frequently reduced. As a result, activated TILs are not able to recognize the tumor-associated antigens and cannot establish an anti-tumor response (30). SCLC also has a high percentage (around 72\%) of FOXP3 regulatory T-cells, which are immune suppressive cells that hamper the anti-tumor response (31).

\section{SCLC immunotherapy trial data}

\section{Checkpoint inhibition}

\section{Previously treated SCLC extensive disease}

For second and higher lines of treatment, results of ICI are generally disappointing, although some patients derive long-term benefit. Currently, third line monotherapy treatment with nivolumab or pembrolizumab (both antiPD-1) is approved by the FDA (no EMA approval) for patients with metastatic SCLC, independent of PD-L1 expression and based on limited data $(32,33)$.

The approval of nivolumab was based on the pooled data from the non-randomized phase $1 / 2$ CheckMate032 trial 
(nivolumab or nivolumab combined with ipilimumab for advanced/metastatic solid tumors) (34). In the randomized SCLC cohort, patients with progression after one or two prior chemotherapy regimens, were treated with nivolumab $3 \mathrm{mg} / \mathrm{kg}$ every 2 weeks $(\mathrm{Q} 2 \mathrm{~W})(\mathrm{N}=147)$ or nivolumab $1 \mathrm{mg} / \mathrm{kg}$ combined with ipilimumab $3 \mathrm{mg} / \mathrm{kg}$ Q3W for four cycles followed by nivolumab $3 \mathrm{mg} / \mathrm{kg}$ Q2W (N=96). Objective response rates (ORR) were $11.6 \%$ and $21.9 \%$, respectively (odds ratio 2.12 ; 95\% CI: $1.06-4.26 ; \mathrm{P}=0.03$ ). This improved ORR did not result in a better OS: median and 24-months OS were 5.7 months (95\% CI: 3.8-7.6) and $17.9 \%$ (nivolumab monotherapy) and 4.7 months (95\% CI: 3.1-8.3) and 16.9\% (nivolumab/ipilimumab). Among the low percentage of patients responding to nivolumab, $61.5 \%$ had a durable response of more than one year, so only a small proportion (about $7 \%$ ) of patients obtains long-term benefit (35). Grade 3-4 treatment related adverse events (TRAEs) were much more common with the immunotherapy combination ( $37.5 \%$ vs. $12.9 \%)$.

Approval of pembrolizumab as a third-line treatment for ED-SCLC was based on the pooled analyses from the phase 1b KEYNOTE-028 (cohort C1, N=24, pembrolizumab $10 \mathrm{mg} / \mathrm{kg}$ ) and the phase 2 KEYNOTE-158 (cohort G, $\mathrm{N}=107$, pembrolizumab $200 \mathrm{mg}$ ) studies $(28,36,37)$. In the KEYNOTE-028, a PD-L1 expression $\geq 1 \%$ was mandatory for enrollment. In KEYNOTE-028, the ORR was 33\%, the median PFS 1.9 months and the median OS 9.7 months. The median DOR was 19.4 months (3.6-20.0+ months). No relationship was shown between efficacy and level of PD-L1 expression (36). In the KEYNOTE-158, 39\% of patients had a PD-L1 expression $\geq 1 \%$. The ORR was $18.7 \%$, the median PFS 2.0 months and the median OS 8.7 months. PD-L1 correlated with improved ORR (35.7\% in PD-L1 positive and $6 \%$ in PD-L1 negative tumors) and improved the OS (14.9 compared to 5.9 months). Twelve patients had DOR $\geq 9$ months (28). In the pooled analyses, 83 patients enrolled had already received $\geq 2$ previous lines, $57 \%$ were PD-L1 positive. The RR and duration of response were $19.3 \%$ and not reached, whereas the median PFS and OS were 2.0 months and 7.7 months, respectively. The grade $\geq 3$ immune-related adverse events (ir-AES) were $6 \%$.

Whether there really exists a benefit of anti-PD1 inhibitors as third-line treatment or whether this benefit is just an over-selection of patients enrolled with good prognostic factors remains unknown, as there is no control arm. In the randomized phase III CheckMate 331 trial however, the efficacy of nivolumab was compared to topotecan or amrubicin as second line treatment (38). The median OS was 7.5 [nivolumab $(\mathrm{N}=284)$ ] versus 8.4 months [chemotherapy $(\mathrm{N}=285)$; hazard ratio (HR) $0.86 ; 95 \%$ confidence interval (CI): 0.72-1.04]. Of note, survival curves in the nivolumab arm are under chemotherapy during the first 12 months, suggesting a potential deleterious effect in outcome with ICI in a subgroup of patients with SCLC. However, in an exploratory analysis, patients with platinum-refractory SCLC, defined as relapse $<90$ days after completion of first-line chemotherapy, nivolumab improved the OS compared with chemotherapy (7.0 vs. 5.7 months, HR 0.71 (95\% CI: 0.54-0.94). AEs were much more common with chemotherapy than with nivolumab: all grade AEs and grade 3-4 TRAEs were seen in 55\% and $4 \%$ of nivolumab treated versus $90 \%$ and $93 \%$ in chemotherapy treated patients.

Atezolizumab (anti-PD-L1) was examined in a phase I trial in 17 patients, from which $\geq 65 \%$ third line (39). Results were poor, with only 1 responder according to RECIST 1.1 $(6 \%)$. Based on immune related response criteria, 4 patients (24\%) responded, and this response was durable; 4 patients $\geq 6$ months, and 2 of these $\geq 12$ months. Median PFS was 1.5 months (95\% CI: $1.2-2.7$ ) and median OS 5.9 months (95\% CI: 4.3-20.1). The most common AE was fatigue in $24 \%$ of the patients. Grade $3-5$ toxicity was seen in 3 patients, including 1 death due to hepatic failure. These results were not confirmed in a subsequent phase II trial. The phase II, non-comparative IFCT1603 trial, randomizing ED-SCLC patients either to atezolizumab $(\mathrm{N}=49)$ or to topotecan $(\mathrm{N}=24)$ in second-line, showed disappointing results. After 6 weeks of treatment, 1 patient responded to atezolizumab $(2.3 \%)$ and disease control rate (DCR) was 20.9\% (95\% CI: 8.8-33.1). Of the chemotherapy treated patients, 2 responded, and DCR was $65 \%$. Median PFS was significantly shorter with atezolizumab (1.4 months, 95\% CI: $1.2-1.5)$ compared to topotecan (4.3 months, $95 \%$ CI: 1.5-5.9) (HR 2.26, $\mathrm{P}=0.04)$. OS was 9.4 months for atezolizumab compared to 11.4 months for chemotherapy (HR $0.84, \mathrm{P}=0.60$ ). Grade 3 toxicity was seen in 20 patients and consisted only of fatigue.

Another anti-PD-L1 antibody, durvalumab, was investigated as monotherapy in a small phase I/II expansion cohort (40). Twenty-one patients with ED-SCLC were treated with durvalumab $10 \mathrm{mg} / \mathrm{kg}$. Partial response (PR) was seen in only 2 patients (9.5\%); however, these responses were durable (14.6 and 29.5+ months). Median PFS was 1.5 months (95\% CI: $0.9-1.8$ ), median OS was 4.8 months (95\% CI: $1.3-10.4$ ) and 12 -month OS rate was $27.6 \%$ (95\% CI: $10.2-48.4)$. AEs were reported in 7 patients (33\%), all 
grade $1-2$.

Durvalumab was also investigated in combination with the anti-CTLA4 antibody tremelimumab for patients with refractory ED-SCLC in the phase II BALTIC study (41). Preliminary results showed 2 out of 21 PR $(9.5 \%)$, and a DCR at 12 weeks of $38 \%$ ( 8 patients). Grade $\geq 3$ AEs were reported in 10 patients $(48 \%)$.

\section{First line SCLC extensive disease}

Based on promising results of two phase II trials $(11,25)$, the phased administration of ipilimumab in addition to chemotherapy was evaluated in a randomized double-blind phase III trial, enrolling 1,132 patients with chemotherapy naïve ED-SCLC, who were randomized to chemotherapy (platinum-etoposide), combined with ipilimumab $10 \mathrm{mg} / \mathrm{kg}$ or placebo (42). Chemotherapy was administered for 4 cycles Q3W, ipilimumab or placebo was added from cycle 3 to 6 Q3W, followed by maintenance ipilimumab or placebo Q12W. The trial was negative for its OS primary endpoint (11.0 vs. 10.9 months; HR 0.94; 95\% CI: 0.81-1.09; $\mathrm{P}=0.377$ ), and no PFS benefit was reported with ipilimumab compared with placebo (HR 0.85; 95\% CI: $0.75-0.97, \mathrm{P}=0.161$ ), with similar toxicity profile (Grade $\geq 3$ TRAE's in $48 \%$ versus $45 \%$, respectively). Diarrhea and colitis were the only grade $\geq 3$ TRAE's that were more frequently seen in patients treated with ipilimumab; $11 \%$ vs. $1 \%$.

Despite these negative results, based on the outcome with anti-PD-L1 in SCLC as monotherapy and the synergism observed with anti-PD(L)-1 and chemotherapy in patients with NSCLC, this strategy was explored in patients with SCLC. The randomized phase III IMpower 133 reported in 403 patients with ED-SCLC that the addition of atezolizumab to standard 4-cycles of etoposidecarboplatin chemotherapy followed by atezolizumab/ placebo maintenance significantly improved the OS over placebo (12.3 vs. 10.3 months, HR 0.70; 95\% CI: 0.54-0.91; $\mathrm{P}=0.007)$ (43). The trial also did achieve the PFS coprimary endpoint (5.2 vs. 4.3 months, HR $0.77,95 \%$ CI: $0.62-0.96 ; \mathrm{P}=0.02)$.

In a recently published survival update after a median follow-up of 22.9 months, the OS benefit remained for atezolizumab although with an increasing HR (12.3 vs. 10.3 months, HR 0.76; 95\% CI: 0.60-0.95; descriptive $\mathrm{P}=0.0154$ ) (44). Of note, survival rate at 18 -months was $13 \%$ higher for atezolizumab compared with placebo $(33.5 \%$ vs. $20.4 \%)$. Patient characteristics associated with longterm survival (living $\geq 18$ months since randomization) were good performance status, $\mathrm{LDH} \leq$ upper limit of normal and sum of longest diameters of the tumor measurements $<$ the median in the total group. Characteristics specifically predictive for atezolizumab benefit could not be identified (45). Importantly, crossover to atezolizumab was not allowed in the trial and only $8 \%$ of patients received ICI at the time of progression in the control arm. Importantly, the addition of atezolizumab did not result in significantly increased toxicity. Moreover, health-related quality of life (HRQoL) as well as physical function, measured by EORTC QLQ-C30 and QLQ-LC13, improved during therapy with a trend of greater improvement with atezolizumab compared to placebo (46). Based on these results, atezolizumab in combination with chemotherapy is approved as a first line treatment for ED-SCLC by FDA and EMA $(47,48)$.

Two other first line phase III trials with the combination of ICI and chemotherapy showed comparable results. The 3-arm CASPIAN trial included 805 patients with treatment-naïve ED-SCLC. Randomization was to platinum-etoposide or platinum-etoposide with durvalumab with or without tremelimumab (anti-CTLA-4) $(49,50)$, stratified by chemotherapy regimen (carboplatin versus cisplatin). In the ICI arms, patients received durvalumab with or without tremelimumab as maintenance treatment in case of no progression to induction treatment. Prophylactic cranial irradiation (PCI) was only allowed in the chemotherapy arm, and crossover was not allowed. Baseline brain metastases were present in $10-14 \%$ of the patients $(10 \%$ for the chemotherapy and chemotherapy plus durvalumab arms, $14 \%$ for the chemotherapy-durvalumabtremelimumab arm), the majority (85-89\%) of the patients with brain metastases had not received brain radiation treatment before study entry. Eight percent of patients in the control group were treated with PCI. In an updated analysis, durvalumab achieved the OS primary endpoint, reporting a median OS of 12.9 months compared with 10.5 months in the control group (HR $0.75 ; 95 \%$ CI: $0.62-0.91 ; \mathrm{P}=0.0032)$ (50). After 18 months, there was an absolute OS benefit of $9 \%$ for durvalumab (32\% vs. $25 \%$ alive). The OS benefit with the addition of durvalumab was similar for the subgroup of patients with brain metastases, compared with the group without brain metastases (51). Interestingly, despite no PCI in the durvalumab arm, the percentage of patients that developed brain metastases was similar to the chemotherapy control arm. The addition of durvalumab did not significantly increase the percentage of $\geq$ grade 3 toxicity. IrAE's were reported in $20 \%$ of patients 
treated with durvalumab, $5 \%$ had grade $\geq 3$. Functioning and HRQoL favoured the combination of durvalumab with chemotherapy compared to chemotherapy alone (52). Durvalumab in combination with chemotherapy is recently approved as a first line treatment for ED-SCLC by the FDA (53). However, the second experimental arm testing tremelimumab in addition to durvalumab and chemotherapy did not meet its primary endpoint of demonstrating a statistically significant improvement in OS in compared with chemotherapy alone (54). The median OS was 10.4 months for tremelimumab and durvalumab in combination with chemotherapy compared with 10.5 months in the control group (HR 0.82; 95\% CI: 0.68-1.00; $\mathrm{P}=0.0451$ ).

An exploratory subgroup analysis assessing a possible relationship between clinical characteristics and outcomes of patients who derived long-term benefit (PFS $\geq 12$ months), showed that $>3$ times more patients treated with durvalumab and chemotherapy derived longterm benefit compared to chemotherapy alone. Clinical characteristics associated with long-term immunotherapy benefit could not be identified (55).

The third first line chemotherapy-ICI combination trial is the KEYNOTE-604 (56), which assessed the survival benefit of adding pembrolizumab versus placebo to standard first-line chemotherapy in 453 patients with ED-SCLC. The addition of pembrolizumab improved the median OS: 10.8 vs. 9.7 months (HR 0.80, 95\% CI: 0.64-0.98, $\mathrm{P}=0.0164)$, however the survival improvement did not meet the prespecified criteria for being considered a positive trial. The co-primary endpoint PFS improved significantly (4.8 vs. 4.3 months, HR 0.75, 95\% CI: 0.61-0.91; $\mathrm{P}=0.0023)$. Toxicities with the addition of pembrolizumab were as expected. HRQoL, measured by EORTC QLQ-C30 and QLQ-LC13, was improved during therapy in both arms, with a trend of greater improvement of cough, chest pain and dyspnea with pembrolizumab compared to placebo.

Of note, patients with an ECOG PS of 2 were excluded from all these phase III trials. The phase IIIb MAURIS trial will hopefully provide an answer whether these patients will also benefit from the addition of ICI to chemotherapy (57). Furthermore, patients with asymptomatic brain metastases are eligible, and thoracic consolidation radiotherapy and PCI are allowed. SPACE (NCT04221529) is a similar trial, including specifically ECOG PS 2 patients.

An overview of the differences in design of the three first line chemo-ICI RCTs is provided in Table 1, outcomes of these trials are presented in Table 2.
Two recently presented phase II trials obtained similar results as the phase III trials mentioned above, and are summarized in Table 2. In contrast to the other trials, in the REACTION trial randomization occurred after 2 cycles of platinum-doublet chemotherapy, and only patients with a response were randomized to chemotherapy and pembrolizumab or chemotherapy alone. By randomizing only responding patients, the benefit of additional immunotherapy could be maximized (58).

\section{Maintenance strategies}

Initially, maintenance therapy with pembrolizumab after induction chemotherapy was investigated in a small single arm phase II trial $(\mathrm{N}=45)$ (59). The median PFS was 1.4 months (95\% CI: $1.3-2.8$ ), with a 1 -year PFS of $13 \%$. The median OS was 9.6 months (95\% CI: 7.0-12.0), with a 1 -year OS of $37 \%$. Afterwards, an exploratory analysis reported that PD-L1 expression $(\geq 1 \%$ on stromal cells, $\mathrm{N}=20$ ) correlated with outcome. Median PFS was 6.8 months for PD-L1 $\geq 1 \%$ compared to 1.3 months, and OS was 12.8 months compared to 7.6 months.

However, maintenance strategy with ICI has not been confirmed in the three-arm randomized phase III CheckMate451 trial. The study enrolled 810 patients and investigated the efficacy of nivolumab with or without ipilimumab $(3 \mathrm{mg} / \mathrm{kg})$ as maintenance therapy after first line induction platinum-etoposide, compared to placebo (60). Patient were stratified by ECOG performance score, prior PCI and sex. Out of patients enrolled, $12-16 \%$ had brain metastases and PCI was performed in $22 \%$ of patients. The primary endpoint was OS for the combination nivolumab and ipilimumab compared to placebo. Nivolumab/ ipilimumab was not superior to placebo (9.2 and 9.6 months, HR 0.92; 95\% CI: 0.75-1.12; $\mathrm{P}=0.3693$ ), with a 1 -year OS rate of $41 \%$ and $40 \%$, respectively. Nivolumab alone did neither result in an OS benefit compared with placebo (10.4 vs. 9.6 months, HR 0.84; 95\% CI: 0.69-1.02), with 1 -year OS $44 \%$ and $40 \%$ respectively. In an OS subgroup analysis, there was an improved OS for patients treated within 5 weeks from last dose of chemotherapy for nivolumab compared to placebo. Remarkably, this benefit was not seen for nivolumab and ipilimumab. Grade $\geq 3$ $\mathrm{AE}$ were reported in 52\% (nivolumab-ipilimumab), $12 \%$ (nivolumab) and $8 \%$ (placebo). The most common grade $\geq 3 \mathrm{AE}$ was diarrhea, respectively $5 \%, 1 \%$ and $0 \%$. Based on these results switch maintenance treatment is not a standard treatment in patients with ED-SCLC.

A new strategy in clinical trials investigating maintenance 
Table 1 Study design of IMpower133, CASPIAN and Keynote-604

\begin{tabular}{|c|c|c|c|}
\hline & IMpower133 & CASPIAN & Keynote-604 \\
\hline $\mathrm{ICl}$ & Atezolizumab & $\begin{array}{l}\text { Durvalumab +/- tremelimumab } \\
\text { (3-arms) }\end{array}$ & Pembrolizumab \\
\hline Phase & III & III & III \\
\hline Median age (years) & 64 & 62 & NA \\
\hline Stratification & Sex, ECOG, brain metastases & Type platinum & Type platinum, ECOG, baseline LDH \\
\hline Imaging & Q6W till week 48, then Q9W & Q6W till week 12, then Q8W & Q6W till week 48, then Q9W \\
\hline Chemo backbone & Etoposide 100 mg/m² & Etoposide $80-100$ mg/m² & Etoposide 100 mg/m² \\
\hline Brain metastases & $\begin{array}{l}\text { Eligible if asymptomatic, } \\
\text { treated, off steroids }\end{array}$ & $\begin{array}{l}\text { Eligible if asymptomatic, treated, off } \\
\text { steroids }\end{array}$ & $\begin{array}{l}\text { Eligible if asymptomatic, treated, off } \\
\text { steroids }\end{array}$ \\
\hline Presence of brain metastases & $17 \%$ & $10-14 \%$ & NA \\
\hline $\mathrm{PCl}$ & Both arms permitted & Only in control arm permitted & Both arms permitted \\
\hline Frequency of $\mathrm{PCl}$ & $10 \%$ & $8 \%$ & NA \\
\hline TRT & Not allowed & Not allowed & Not allowed \\
\hline Treatment beyond PD & If clinical benefit & If clinical benefit & Permitted at investigators discretion \\
\hline
\end{tabular}

NA, not available.

therapy is the IMPULSE study. In this trial, the efficacy and safety of the Toll-like 9 receptor-agonist lefitolimod was investigated as a maintenance treatment after induction platinum based chemotherapy (61). No improvement for PFS or OS was found in the whole population, however two predefined patient subgroups showed promising results favoring lefitolimod: patients with a low frequency of activated CD86+ B-cells (HR 0.53, 95\% CI: 0.26-1.08) and patients with reported chronic obstructive pulmonary disease (COPD) (HR 0.48, 95\% CI: 0.20-1.1).

\section{Radiotherapy}

Based on the survival benefit found in the PACIFIC trial (adjuvant durvalumab in stage III NSCLC patients treated with concurrent chemoradiotherapy) (13), the addition of ICI to chemoradiation in SCLC is of interest and several trials are ongoing.
Radiotherapy combined with ICI holds promise for subgroups in stage IV NSCLC, as was shown for instance in the Pembro-RT study (62). This potential role of thoracic radiotherapy in a concurrent setting with immunotherapy after upfront chemotherapy, was investigated in a phase I/II trial with nivolumab $1 \mathrm{mkg} / \mathrm{kg}$ and ipilimumab $3 \mathrm{mg} / \mathrm{kg}(63)$ in SCLC. 21 patients with ED-SCLC were treated with 4-6 cycles platinum-etoposide, followed by combination treatment with thoracic radiotherapy (10 fractions, total of 30 Gy), and ICI (nivolumab and ipilimumab). The 6 months PFS was 24\% (95\% CI: 9-43\%), which is similar to historical data. This was the reason to discontinue this trial early. The median estimated PFS was 4.5 months $(95 \%$ CI: 2.7-4.6) and the median estimated OS was 11.7 months (95\% CI: 4.7-16.0).

A comparable phase I trial was performed with pembrolizumab in combination with thoracic radiotherapy after induction chemotherapy for ED- 
Table 2 Outcomes of IMpower133, CASPIAN and Keynote-604

\begin{tabular}{|c|c|c|c|c|c|c|c|c|c|c|c|c|}
\hline & \multicolumn{5}{|c|}{ Median OS (months) } & \multicolumn{5}{|c|}{ Median PFS (months) } & \multicolumn{2}{|c|}{ 18-months OS } \\
\hline IMpower133 & 12.3 & 10.3 & 0.76 & $0.60-0.95$ & 0.0154 & 5.2 & 4.3 & 0.77 & $0.62-0.96$ & 0.02 & $33.5 \%$ & $20.4 \%$ \\
\hline CASPIAN (D) & 12.9 & 10.5 & 0.75 & $0.62-0.91$ & 0.0032 & 5.1 & 5.4 & 0.80 & $0.66-0.96$ & Unknown & $32 \%$ & $25 \%$ \\
\hline CASPIAN $(D+T)$ & 10.4 & 10.5 & 0.82 & $0.68-1.00$ & 0.0451 & 4.9 & 5.4 & 0.84 & $0.70-1.01$ & Unknown & & \\
\hline
\end{tabular}

$\mathrm{D}$, durvalumab; T, tremelimumab; NA, not available.

SCLC. Thirty-eight patients were treated with 16 cycles pembrolizumab every 3 weeks combined with 45 Gy thoracic radiotherapy in 15 daily fractions. Median PFS was 6.1 months (95\% CI: 4.1-8.1) and the median OS 8.4 months (95\% CI: 6.7-10.1) (64).

In conclusion, in line with the results of maintenance systemic treatments in stage IV disease, thoracic radiotherapy with ICI after induction chemotherapy failed to improve the outcome. The role of radiotherapy concurrent with ICI after induction chemo-immunotherapy is currently being investigated in the phase II-III RAPTOR trial (NCT04402788). Furthermore, several trials are ongoing in LD-SCLC, concurrent with chemoradiotherapy [phase II-III NRG-LU005 (NCT03811002), N=506, phase II trial (NCT03585998), N=51] or after concurrent chemoradiotherapy [phase II STIMULI (NCT02046733), $\mathrm{N}=174$, phase II ACHILES study (NCT03540420), $\mathrm{N}=212$, phase III ADRIATIC study (NCT03703297), $\mathrm{N}=600]$. In addition, several comparable phase I trials are ongoing. Only for the STIMULI trial, results have been reported (65). 153 patients with LD-SCLC were treated with 4 cycles of chemotherapy with concurrent radiotherapy followed by PCI. Non-progressing patients were randomized between adjuvant immunotherapy (nivolumabipilimumab) or observation. The primary endpoint, median PFS, was 10.7 months for nivolumab and ipilimumab compared to 14.5 months for observation (HR 1.02, 95\% CI: $0.66-1.58, \mathrm{P}=0.93)$. Of note, the median time to discontinuation of treatment was 1.7 months for nivolumab and ipilimumab, of which $55 \%$ was due to toxicity.

\section{Vaccination trials}

Another way to induce an immune response is by using tumor vaccines. These vaccines can elicit an in vivo immune response specifically toward tumor-associated antigens formulated in the vaccine, or by directly administering antigen-stimulated T cells or dendritic cells (DCs). Tumor vaccines have already been shown to be promising and safe in NSCLC, however depending on the type of vaccine (66). Cellular vaccination was found to be more active then peptide vaccination. Several trials using vaccinations were performed in SCLC or are still ongoing.

A randomized phase III trial in LD-SCLC investigated whether Bec2/bacille Calmette-Guerin (BCG) vaccination prolongs survival in patients with LD-SCLC responding to chemoradiotherapy (67). Bec2 is an anti-idiotypic antibody that mimics GD3, which is expressed on the surface of tumor cells. Five hundred and fifteen patients were randomized between five vaccinations or observation. This study did not meet its primary endpoint (OS). Median OS was 14.3 months for vaccination compared to 16.4 months for the observation group (HR 1.12, 95\% CI: 0.91-1.37, $\mathrm{P}=0.28)$. Median PFS was 5.7 months for vaccination compared to 6.3 months for observation (HR 1.11, 95\% CI: $0.95-1.36, \mathrm{P}=0.30)$.

Another strategy is personalized peptide vaccination (PPV), in which vaccine antigens were selected based on pre-existing host immunity (68). PPV was tested in 10 patients who failed to respond to chemotherapy with or without radiotherapy. Patients were vaccinated weekly for six consecutive weeks and then bi-weekly thereafter. In four patients, PPV was discontinued during the weekly vaccination due to rapid disease progression. The other six patients experienced a peptide-specific immunological boosting. Four patients had a survival of $25,9.5,6.5$ and 6 months and 2 patients were still alive at data base lock (survival 24.5 and 10 months).

A comparable phase II trial of PPV was performed in 46 pretreated and patients with treatment-naïve EDSCLC (69). Seventy percent of patients had IgG responses to the vaccinated peptides after 1 vaccination cycle, $95 \%$ of patients after 2 cycles. Median OS was significantly improved in patients with augmented IgG responses to a 
greater number of nonvaccinated peptides after the second cycle of vaccination $(1,237 v s .382$ days, $\mathrm{P}=0.010)$.

Two phase I trials were performed with vaccinations administered to patients with LD-SCLC as well as EDSCLC who had a major response to first line chemotherapy. One of these trials tested the immunogenicity of three different doses of a synthetic version of ganglioside fucosyl-GM1-KLH conjugate (70). Vaccination was found to be safe an induced an IgM-antibody response. The other trial was using vaccination with polysialic acid (polySA) (71). PolySa is a polymer side chain bound to the neural cell adhesion molecule that is extensively expressed on the surface of SCLC cells. This vaccination also was found to be safe and resulted in higher antibody responses.

Another trial investigated a vaccine consisting of DC transduced with the full-length wild-type p53 gene delivered via an adenoviral vector in 29 patients with EDSCLC (72). P53 is mutated in approximately $90 \%$ of SCLC. In this trial, $57.1 \%$ of the patients had $\mathrm{p} 53$-specific $\mathrm{T}$ cell responses to the vaccination. However, only 1 patient showed a clinical response. Interestingly, $61.9 \%$ of patients responded to chemotherapy that immediately followed vaccination. These responses were associated with induction of immunologic response to vaccination, which suggests that more effective treatment results are possible by optimal use of vaccination combined with chemotherapy.

In conclusion, to date, the only phase III trial evaluating a tumor vaccine in SCLC has failed to improve OS, other trials are small and benefit of vaccines has not been established. It seems difficult to stimulate the response of the patients' immune system using vaccine therapy. However, the use of a combination of therapies might enhance the effect of tumor vaccines.

\section{Trials using interferon}

Interferon (IFN) is the first discovered cytokine with efficacy on cancer cells (73). It was first used in natural form, followed by using in recombinant form. IFN is released by host cells in response to viral stimulation and can activate immune cells, such as macrophages and natural killer cells. In addition, IFN upregulates major histocompatibility complex (MHC) antigens leading to an increased antigen presentation. Furthermore, IFN suppresses angiogenesis and suppresses the proliferation of endothelial cells leading to a decreasing tumor growth.

In the early 1980s, recombinant IFN was investigated in combination with chemotherapy in SCLC patients, with the aim to prevent early relapse after chemotherapy, after in vitro studies have shown a durable effect by using IFN in combination with chemotherapy (74). These studies showed modest or no improvement in survival $(75,76)$.

In 2013 , the effect of interferon combined with chemotherapy was evaluated in a randomized phase II trial with 164 patients (77). Patients were treated with chemotherapy alone, a combination of chemotherapy with IFN-alpha, IFN-gamma or IFN-alpha and IFN-gamma. Median survival was 10 months for chemotherapy alone (95\% CI: 9.3-10.6), 10.3 months for chemotherapy with IFN-alpha (95\% CI: 7.13-13.5), 8.3 months for IFNgamma (95\% CI: 6.8-9.8 months), and 11 months for IFN-alpha and IFN-gamma (95\% CI: 9.2-12.8 months), concluding no significant difference among all four groups. However, looking to only patients with LD-SCLC, a significant survival benefit was found for chemotherapy with IFN-alpha, showing a median OS of 34 months compared to 19 months for chemotherapy alone $(\mathrm{P}=0.039)$, 13.6 months for INF-gamma $(\mathrm{P}=0.005)$ and 17 months for IFN-alpha and IFN-gamma $(\mathrm{P}=0.038)$. IFN remains a potential auxiliary therapy in patients with SCLC, and further trials are needed to identify its effect.

\section{Trials using novel agents}

Another promising strategy is the use of antibodies against tumor-associated antigens. Ganglioside fucosyl-GM1 (FucGM1) is expressed in SCLC but absent in most normal tissues. Preclinically, the addition of anti-PD-1 or antiCD137 to BMS-986012 (FucGM1 antibody) improved the therapeutic efficacy of BMS-986012 significantly (78). BMS-986012 showed early promising safety and efficacy in pretreated SCLC patients when combined with nivolumab.

Stimulating the innate immune system is also an attractive option. Phagocytosis of SCLC cells by macrophages is inhibited by CD47 expression on the SCLC. Preclinically, anti-CD47 resulted in SCLC tumor responses (79). Phase I trials including patients with solid malignancies are ongoing, also with the addition of pembrolizumab.

\section{Biomarkers}

With the FDA approval of PD-(L)1 ICI in combination with chemotherapy as a first line treatment in ED-SCLC and PD-1 ICI as a third line option in ED-SCLC, we have made an important advance in treating SCLC patients, whose treatment options have remained unchanged for 
decades. However, critical analysis of these trials shows that only a small part of the patients benefits from ICI. Identifying these patients is a real clinical challenge.

Several biomarkers have been investigated or are currently being investigated. The most evaluated biomarkers are PD-L1 and TMB.

\section{$P D-(L) 1$}

PD-1 is a receptor expressed on the surface of T-cells regulating T-cell activation and proliferation. Its ligand $\mathrm{PD}-$ L1 is (over)expressed on various tumor cells. In contrast to NSCLC, where PD-L1 expression is used in deciding which therapy is preferred, the clinical relevance of PD-L1 expression in SCLC has remained unclear.

\section{PD-L1 expression on tumor cells}

In the Checkmate032, PD-L1 expression [tumor cells (TC)] was evaluated in 148 patients with the $28-8$ pharmDx antibody (80). PD-L1 expression was found to be positive $\approx 10 \%$ of cases, negative in $\approx 60 \%$ and unknown in $\approx 30 \%$. No significant association was found between PDL1 expression and ORR for nivolumab or combination nivolumab with ipilimumab.

\section{PD-L1 expression on tumor or immune cells}

Of the 403 included patients in the IMpower133 trial, only 137 patients had evaluable tumor material, which reflects the difficulty in obtaining biopsies in SCLC. PD$\mathrm{L} 1$ expression was analyzed on TC as well as on immune cells (IC) with the VENTANA SP263 antibody (44). 129 patients $(94.2 \%)$ had PD-L1 TC expression $<1 \%$ and 68 patients $(49.6 \%)$ had PD-L1 IC expression $<1 \%$. In subgroup analyses, an inverse correlation was found between PD-L1 expression on TC or IC and OS: a negative PD-L1 expression appeared to be predictive for a better OS (median OS 10.2 months for combination chemotherapy with atezolizumab versus 8.3 months for chemotherapy with placebo, HR $0.51,95 \%$ CI: $0.30-0.89$ ). This result is inconsistent with previous data and needs further analysis.

\section{Combined PD-L1 positive score (CPS)}

CPS has not yet been evaluated in first line therapy, only in relapsed SCLC. CPS is the sum of the number of PD-L1stained cells, such as TC, lymphocytes and macrophages), divided by the total number of viable TC, multiplied by 100 (81). The maximum score is defined as 100 .

CPS was evaluated in the KEYNOTE-028 trial and in the KEYNOTE-158. In the KEYNOTE-028, CPS $\geq 1 \%$ was an inclusion criterion for treatment with pembrolizumab. ORR was $33 \%$ (8 of 24) (36). The KEYNOTE-158 was stratifying patients into a CPS $\geq 1 \%$ ( $\mathrm{n}=48)$ subgroup and a CPS $<1 \%(\mathrm{n}=50)$ subgroup. Results in de CPS $\geq 1 \%$ subgroup where an ORR of $35.7 \%$, a 1 year OS of $53.1 \%$ and a median OS duration 14.6 months, compared to respectively $6 \%, 30.7 \%$ and 7.7 months for the CPS $<1 \%$ subgroup. Among patients with unknown PD-L1 status, ORR was $27 \%$ (4 of 15) (28).

In conclusion, no correlation was found between PDL1 expression and efficacy in SCLC. However, looking to KEYNOTE-158 using combined PD-L1 score, CPS might to have predictive value.

\section{Tumor mutational burden}

TMB is usually defined as the number of somatic mutations found in the DNA of cancer cells per megabase (Mb) (82). Determination of TMB can be done by several DNA sequencing methods, of which Whole Exome Sequencing (WES) is considered the gold standard. Some trials are using targeted next-generation sequencing (NGS) panels to extrapolate TMB, which leads to a variation in thresholds for TMB and reproducibility of TMB. This causes interassay variation, which limits the utility of panel-based TMB. Therefore, WES remains the most comprehensive, reproducible and reliable method to determine TMB (83).

TMB was evaluated in the CheckMate032 using WES. The tertiles were defined as $<143$ mutations (low), 143-247 mutations (intermediate) and $\geq 248$ mutations (high). Of all treated patients, $61 \%$ had sufficient paired tumor and whole blood samples for WES. In $86 \%$ of these patients WES could be performed successfully: overall 211 (53\%) of all treated patients were evaluable for efficacy analyses by TMB (80). In the Checkmate032, ORR was $5 \%$ in the TMB low subgroup $(\mathrm{n}=42), 7 \%$ in the intermediate subgroup $(\mathrm{n}=44)$ and $21 \%$ in the TMB high subgroup $(\mathrm{n}=47)$. Median PFS was 1.3, 1.3 and 1.4 months, median OS was 3.1, 3.9 and 5.4 months and 1-year OS were 22\%, 26\% and $35 \%$ respectively (80). For the patients treated with combination nivolumab $1 \mathrm{mg} / \mathrm{kg}$ and ipilimumab $3 \mathrm{mg} / \mathrm{kg}$, results were comparable, showing ORR of respectively $22 \%, 16 \%$ and $46 \%$, median PFS of 1.5 months, 1.3 months and 7.8 months, median OS 3.4, 3.6 and 22 months and 1-year OS of $23 \%, 20 \%$ and $62 \%$.

The KEYNOTE-028 was also evaluating TMB in multiple tumor types. However, in this basket trial 
consisting of 471 patients in total, TMB data were available for 77 patients, and of these only 4 patients had SCLC (84). For all tumor types, higher TMB was associated with higher ORR $(\mathrm{P}=0.018)$ and longer PFS $(\mathrm{P}=0.051)$. The KEYNOTE-158, a basket trial consisting of 1032 patients, contained 751 patients with evaluable TMB in multiple tumor types. Of these, 75 patients had SCLC. The RR for patients with higher TMB was $29 \%$ (10 out of 34 patients) compared to $10 \%$ for patients with low TMB (4 out of 41 patients) (85).

In conclusion, TMB may be useful to predict benefit from immunotherapy in SCLC. However, the sample size is too limited to draw firm conclusions and the difficulties to encounter when using TMB, makes TMB as a challenging and not preferable biomarker in practice.

\section{Blood-based tumor mutational burden}

WES using tumor tissue has several disadvantages, for example the need of tumor biopsies, the time-consuming analysis and the costs. BTMB is measured by targeted NGS using cell-free DNA (cfDNA) (86). Using NGS on blood instead might overcome the previously stated problems.

The IMpower133 investigated the correlation between bTMB and OS (43). 394 cancer-associated genes were assessed by NGS. Two cut-offs of bTMB were used: $10 \mathrm{mut} / \mathrm{Mb}$ and $16 \mathrm{mut} / \mathrm{Mb}$. Using a cut-off of $<10 \mathrm{mut} / \mathrm{Mb}$ $(\mathrm{n}=139)$, median OS was 11.8 months for combination chemotherapy and atezolizumab compared to 9.2 months for chemotherapy alone (HR 0.70, 95\% CI: 0.45-1.07). For patients with $\mathrm{bTMB}$ of $\geq 10 \mathrm{mut} / \mathrm{Mb} \quad(\mathrm{n}=212)$, median OS was respectively 14.6 months compared to 11.2 months (HR 0.68, 95\% CI: 0.47-0.97).

A similar improvement in OS was found by using another cut-off of $16 \mathrm{mut} / \mathrm{Mb}$ : for patients with a bTMB $<16 \mathrm{mut} / \mathrm{mB}$, median OS was 12.5 months for combination chemotherapy and atezolizumab compared to 9.9 months for chemotherapy alone (HR 0.71, 95\% CI: 0.52-0.98). For patients with a bTMB of $\geq 16$ mut/Mb, median OS was 17.8 months compared to 11.9 months respectively (HR $0.63,95 \%$ CI: $0.35-1.15$ ).

Because the different cut-off subgroups are showing similar improvements in OS, bTMB seems not to be a useful predictive biomarker.

\section{Future directions}

Although the outcome of patients with SCLC has improved by adding ICI to standard first line chemotherapy, and durable responses have been observed with ICI as monotherapy in third line, the majority of the patients do not benefit. Better patient selection is needed, as well as new combinations of drugs. Furthermore, the role of PCI should be redefined in the ICI era. Possible future directions are discussed below.

\section{Future directions for biomarkers}

The immune contexture of SCLC is often an immuneexcluded, non-inflamed T-cell environment (87-89). Furthermore, SCLC cells often express CD47 $(79,90)$, which protects the cells from phagocytosis by macrophages and dendritic cells. Moreover, TILs often express coinhibitory immune checkpoint proteins such as TIM3, LAG3 and FOXP3 (91).

Full characterization of the immune environment is challenging due to the difficulty of obtaining enough tissue and subsequently analyzing this tissue in patients that often have a rapidly deteriorating clinical condition. To date, there are no useful predictive biomarkers to select the subgroup of patients that might have long term benefit from immunotherapy. A recent multicenter retrospective analyses suggested a correlation between irAEs and response to ICI (92). In 183 patients treated with antiPD-(L)1 (59.6\%) with or without a CTLA-4 inhibitor, irAEs were reported in $39.9 \%$. The ORR of patients who experienced at least one irAE was $27.4 \%$, compared to $3.6 \%$ for patients without irAEs. Furthermore, the median PFS was 3.8 and 1.3 months respectively, the median OS 13.8 and 2.9 months. These results were adjusted for age, sex, performance status and presence of brain metastases and need to be prospectively validated.

Biopsies upon progression on ICI are also needed in order to optimize treatment for patients with SCLC. Hopefully the REBIMMUNE trial (NCT04300062) will elucidate some of these mechanisms.

It would also be interesting to evaluate the value of serial circulating tumor DNA (ctDNA) measurements as monitoring option for patients with SCLC treated with ICI. Indeed, in small series, a decrease in ctDNA has been associated with platinum-sensitivity in $\operatorname{SCLC}(93,94)$. Although data exists for NSCLC (95), data for monitoring of ICI efficacy in SCLC are very limited. In a small series $(\mathrm{N}=27$ patients with SCLC, 8 receiving ICI), monitoring by using plasma cell-free DNA (cfDNA) has been described (96). An increase in cfDNA could be identified 
before the occurrence of radiological progression. If validated, this could provide a non-invasive option for treatment monitoring.

\section{Future directions for new combinations of drugs}

Due to the immune-excluded environment of SCLC, the combination of other treatments with immunotherapy seems logical to convert SCLC to an immunogenic tumor. Several options are described below. A summary of all ongoing trials can be found at https://cdn.amegroups.cn/ static/public/TLCR-20-630-1.pdf.

(Thoracic) radiotherapy is a logical option to add to ICI, or other immune modifying drugs as radiation acts synergistically with immunotherapy (97). Unfortunately, as described above, TRT (45 Gy) combined with ICI, did not improve outcomes $(59,64)$. Moreover, median OS was disappointing with 8.4 months, and similar to the CRESTtrial (98). Furthermore, adjuvant nivolumab-ipilimumab failed to improve OS for patients with LD-SCLC treated with chemoradiation (65). Results from other trials are awaited.

SCLC is very vulnerable to DNA damage; therefore, DNA damage repair inhibitors (like PARP inhibitors) and cell cycle checkpoint kinase inhibitors (inhibition of for example CHK1, WEE1, aurora kinase A (AURKA), cyclindependent kinase 7 (CDK7) are of interest (99). However, results in unselected SCLC patients using monotherapy or combination therapies with chemotherapy were generally disappointing (100). Combinations with immunotherapy are currently tested preclinically or in early phase trials.

PARP inhibition results in STING pathway activation, interferon signaling and enhancement of Tcel CD4/ CD8 infiltration, at least in vivo (101). Unfortunately, a phase II trial evaluating the combination of olaparib and durvalumab in relapsed SCLC $(\mathrm{N}=20)$ was negative (102). It could be that more potent PARP inhibitors such as talazoparib or niraparib, or PARP inhibition combined with CHK1 inhibition together with an ICI can obtain better outcomes (101).

Preclinically and in breast cancer patients, cyclindependent kinase 4/6 (CDK4/6) inhibition with abemaciclib or palbociclib resulted in enhanced anti-tumor immunity, by increasing the functional capacity of tumor cells to present antigens and by reducing the proliferation of Tregs (103). CDK4/6 inhibitors are also investigated in SCLC.

Selective CDK7 inhibition (with YKL-5-124) is also promising, as besides inducing DNA replication stress and genomic instability, it also induces immune response signaling (103). The combination with anti-PD-1 is being tested preclinically.

Lurbinectedin, targeting the enzyme RNA polymerase II, and inducer of DNA double-strand breaks, showed promising activity in phase I and II trials, either as monotherapy or combined with doxorubicin (104-106). Interestingly, in preclinical models, lurbinectedin acted synergistically with ICI, and reduces tumor associated macrophages $(107,108)$. The combination of lurbinectedin with atezolizumab is being investigated in SCLC in a phase I trial.

Instead of checkpoint blockade, co-stimulation of T-cell responses with monoclonal antibody agonists is also being explored. Several trials are ongoing with or without combination with ICI and/or chemotherapy.

\section{Future directions for selection of patients}

As is described above, multiple combination therapies are possible, and it is challenging to select the most promising treatments, and to select the right SCLC patient for each treatment.

In a recently published paper, Rudin et al. subdivided SCLC into different neuroendocrine subtypes which can be distinguished based on the expression of four key transcriptional regulators: ASCL1 (=ASH1), NEUROD1, POU2F3 and YAP1 (99). Four subtypes were described: The first two are markers of the SCLC neuroendocrine subtypes, the latter two of the non-neuroendocrine ones. The most common (70\%) is the "classical" SCLC (SCLC-A) which is characterized by ASCL1 expression. Regarding genomic profile, this subtype has both TP53 and RB1 loss. On a transcriptional level, SCLC-A is characterized by high ASCL1, INSM1, L-MYC and DLL3 expression, and low NEUROD1 expression. Immunohistochemically, SCLC-A is TTF-1 high and C-MYC low. The other neuroendocrine variant (11\% of cases) is characterized by NEUROD1 expression and is called SCLC-N. Besides NEUROD1 expression, it differs from SCLC-A in ASCL1 expression (variable) and TTF-1 and C-MYC expression (low and high, respectively). The most common non-neuroendocrine (16\% of SCLC cases, SCLC-P) variant is characterized by POU2F3 expression. It further differs from the SCLC-A variant regarding ASCL1 and INSM1 expression (low). The last variant (SCLC-Y) is rare (3\%) and is characterized by YAP1 expression. It is RB1 wildtype/enriched, ASCL1, NEUROD1 and ISM1 low (99). 
It might be that different subtypes have different immune environments. For example, in a small series of 61 cases of SCLC and pulmonary carcinoids, those with SCLC-Y were enriched for a T-cell inflamed phenotype (109). These subtypes might be used in the future to select (immune) therapies most suitable for combination with PD-(L)1inhibitors.

For example, DLL3 expression is high only in SCLC-A. Although theoretically interesting, DLL3 inhibition alone with Rova-T failed to meet the prespecified endpoints in the phase II TRINITY trial including pretreated DLL3 expressing SCLC (110). It could be interesting to combine Rova-T with ICI in DLL3 high patients. In a phase I/II study ( $\mathrm{N}=42)$ Rova- $\mathrm{T}$ was combined with nivolumab plus ipilimumab $(\mathrm{N}=12)$ or nivolumab alone $(\mathrm{N}=30)$. The nivoipi group was prematurely closed due to toxicity, and also Rova-T plus nivolumab was toxic $(53 \% \geq$ grade 3 toxicity of which $10 \%$ grade 5). Furthermore, ORR was $22 \%$ in the Rova-T nivolumab group with a disappointing median DOR of 3.8 months (111). Based on the negative MERU and TAHOE studies, the Rova-T program is now discontinued. Bispecific T-cell engagers and chimeric antigen receptor (CAR) T-cell constructs are currently being investigated. The use of these drugs seems most interesting in DLL3 high patients (SCLC-A subtype), but in both phase I trials, no selection is performed based on DLL3.

As another example, the combination of PARP with antiPD-L1 inhibition preclinically seems especially promising in SCLC-A (101).

An SVV oncolytic virus has selective tropism for SCLC-N (112). Furthermore, it seems that MYC-high SCLC is especially sensitive to aurora kinase inhibitors such as alisertib (113). SCLC-P seems most vulnerable to IGF1R inhibition (114). However, trial data need to be awaited.

\section{Future directions for PCI}

The role of PCI has been questioned with the results of a Japanese phase III trial, in which SCLC patients were randomized between PCI and follow-up with brain MRI (115). A currently ongoing phase III trial is evaluating whether MRI combined with PCI is not inferior tot MRI surveillance alone (NCT04155034), for patients (LD-SCLC as well as ED-SCLC) that have competed their first line treatment (ICI to the discretion of the physician). In NSCLC, it has been suggested that PD-(L)1 inhibition can prevent brain metastases development $(13,116)$. For SCLC, no data exist to the best of our knowledge, and whether there is a role of PCI in a chemo-ICI regimen should be further evaluated. Furthermore, it is not well known whether PCI can be given safely concurrent with PD-(L) 1 inhibition, as only 22 out of the 198 patients in the IMpower133 trial randomized to atezolizumab, were treated with PCI concurrently with atezolizumab and detailed neurotoxicity data have not been reported. However, no grade 3-5 neurological adverse events were reported for the total atezolizumab group.

Numerous retrospective case series in several tumor types suggest that combination of ICI with cranial radiotherapy is safe, however prospective studies are needed to further confirm these findings (117).

\section{Conclusions}

SCLC is a disease with a poor prognosis. Even though the incidence of SCLC is decreasing, there is a need for more effective treatment opportunities. With the recent EMA approval of atezolizumab and FDA approval of also durvalumab in combination with chemotherapy as a first line treatment in ED-SCLC and additional FDA approval for nivolumab/pembrolizumab as a second/third line option in ED-SCLC, we have made an important step in treating patients with SCLC. However, only a small part of the patients benefits from ICI. Numerous studies are currently being performed aiming to improve ICI benefit in SCLC, for example with the addition of radiotherapy, co-stimulatory antibodies, and other immune modifying agents. Prospective trials should include biomarker research and consider the neuroendocrine subtyping of SCLC in order to select patients most likely to benefit.

\section{Acknowledgments}

Funding: None.

\section{Footnote}

Provenance and Peer Review: This article was commissioned by the editorial office, Translational Lung Cancer Research for the series "Immunotherapy in other thoracic malignancies and uncommon populations". The article has undergone external peer review.

Reporting Checklist: The authors have completed the Narrative Review reporting checklist. Available at http:// dx.doi.org/10.21037/tlcr-20-630 
Conflicts of Interest: All authors have completed the ICMJE uniform disclosure form (available at http://dx.doi. org/10.21037/tlcr-20-630). The series "Immunotherapy in other thoracic malignancies and uncommon populations" was commissioned by the editorial office without any funding or sponsorship. JGJV Aerts serves as an unpaid editorial board member of Translational Lung Cancer Research from Sep 2019 to Sep 2021. JR served as the unpaid Guest Editor of the series and serves as an unpaid editorial board member of Translational Lung Cancer Research from Sep 2019 to Sep 2021. DD reports personal fees from Roche, personal fees from BMS, personal fees from MSD, personal fees from Pfizer, personal fees from Astra Zeneca, personal fees from Novartis, outside the submitted work. AMD reports other from Roche, other from BOEHRINGER, other from Pharmamar, other from Pfizer, other from Takeda, grants and other from BMS, other from Lilly, grants from Abbvie, outside the submitted work. JGJVA reports personal fees and non-financial support from msd, personal fees from bms, personal fees from boehringer ingelheim, personal fees from amphera, personal fees from eli-lilly, personal fees from takeda, personal fees from bayer, personal fees from roche, personal fees from astra zeneca, outside the submitted work; In addition, Dr. JGJVA has a patent allogenic tumor cell lysate licensed to amphera, a patent combination immunotherapy in cancer pending, and a patent biomarker for immunotherapy pending. JR reports other from MSD, other from Boehringer, other from Pfizer, personal fees and other from Ose Immunotherapeutics, other from BMS, other from AstraZeneca, other from Roche, outside the submitted work. DDR reports grants from Boehringer, other from Celgene, grants and other from BMS, other from Pfizer, other from Roche, grants and other from AstraZeneca, other from MSD, other from Seattle genetics, grants from Philips, grants from Olink, outside the submitted work. LH reports grants and other from Roche, grants and other from Boehringer, grants from AstraZeneca, other from BMS, other from Eli Lilly, other from Pfizer, other from Takeda, other from MSD, from null, outside the submitted work. The authors have no other conflicts of interest to declare.

Ethical Statement: The authors are accountable for all aspects of the work in ensuring that questions related to the accuracy or integrity of any part of the work are appropriately investigated and resolved.

Open Access Statement: This is an Open Access article distributed in accordance with the Creative Commons Attribution-NonCommercial-NoDerivs 4.0 International License (CC BY-NC-ND 4.0), which permits the noncommercial replication and distribution of the article with the strict proviso that no changes or edits are made and the original work is properly cited (including links to both the formal publication through the relevant DOI and the license). See: https://creativecommons.org/licenses/by-nc-nd/4.0/.

\section{References}

1. de Groot PM, Wu CC, Carter BW, Munden RF. The epidemiology of lung cancer. Transl Lung Cancer Res 2018;7:220-33.

2. Ou SH, Ziogas A, Zell JA. Prognostic factors for survival in extensive stage small cell lung cancer (ED-SCLC): the importance of smoking history, socioeconomic and marital statuses, and ethnicity. J Thorac Oncol 2009;4:37-43.

3. Malik SM, Korn E, Thomas A. Small cell lung cancer: Why has it become an orphan disease? J Clin Oncol 2015;33:7578.

4. Lekic M, Kovac V, Triller N, et al. Outcome of small cell lung cancer (SCLC) patients with brain metastases in a routine clinical setting. Radiol Oncol 2012;46:54-9.

5. Lung Cancer Survival Rates I 5-Year Survival Rates for Lung Cancer [Internet]. [cited 2020 Feb 10]. Available online: https://www.cancer.org/cancer/lung-cancer/ detection-diagnosis-staging/survival-rates.html

6. Lim W, Ridge CA, Nicholson AG, et al. The 8th lung cancer TNM classification and clinical staging system: review of the changes and clinical implications. Quant Imaging Med Surg 2018;8:709-18.

7. De Ruysscher D, Lueza B, Le Péchoux C, et al. Impact of thoracic radiotherapy timing in limited-stage small-cell lung cancer: usefulness of the individual patient data metaanalysis. Ann Oncol 2016;27:1818-28.

8. Mak DWS, Li S, Minchom A. Challenging the recalcitrant disease-developing molecularly driven treatments for small cell lung cancer. Eur J Cancer 2019;119:132-50.

9. Rudin CM, Ismaila N, Hann CL, et al. Treatment of Small-Cell Lung Cancer: American Society of Clinical Oncology Endorsement of the American College of Chest Physicians Guideline. J Clin Oncol 2015;33:4106-11.

10. von Pawel J, Schiller JH, Shepherd FA, et al. Topotecan versus cyclophosphamide, doxorubicin, and vincristine for the treatment of recurrent small-cell lung cancer. J Clin Oncol 1999;17:658-67.

11. Reck M, Bondarenko I, Luft A, et al. Ipilimumab in 
combination with paclitaxel and carboplatin as first-line therapy in extensive-disease-small-cell lung cancer: results from a randomized, double-blind, multicenter phase 2 trial. Ann Oncol 2013;24:75-83.

12. Paz-Ares L, Luft A, Vicente D, et al. Pembrolizumab plus Chemotherapy for Squamous Non-Small-Cell Lung Cancer. N Engl J Med 2018;379:2040-51.

13. Antonia SJ, Villegas A, Daniel D, et al. Overall Survival with Durvalumab after Chemoradiotherapy in Stage III NSCLC. N Engl J Med 2018;379:2342-50.

14. Gandhi L, Rodríguez-Abreu D, Gadgeel S, et al. Pembrolizumab plus Chemotherapy in Metastatic NonSmall-Cell Lung Cancer. N Engl J Med 2018;378:2078-92.

15. Horn L, Spigel DR, Vokes EE, et al. Nivolumab Versus Docetaxel in Previously Treated Patients With Advanced Non-Small-Cell Lung Cancer: Two-Year Outcomes From Two Randomized, Open-Label, Phase III Trials (CheckMate 017 and CheckMate 057). J Clin Oncol 2017;35:3924-33.

16. Ribas A, Wolchok JD. Cancer immunotherapy using checkpoint blockade. Science 2018;359:1350-5.

17. Chen DS, Mellman I. Oncology meets immunology: the cancer-immunity cycle. Immunity 2013;39:1-10.

18. George J, Lim JS, Jang SJ, et al. Comprehensive genomic profiles of small cell lung cancer. Nature 2015;524:47-53.

19. Alexandrov LB, Nik-Zainal S, Wedge DC, et al. Signatures of mutational processes in human cancer. Nature 2013;500:415-21.

20. Goodman AM, Kato S, Bazhenova L, et al. Tumor Mutational Burden as an Independent Predictor of Response to Immunotherapy in Diverse Cancers. Mol Cancer Ther 2017;16:2598-608.

21. Yarchoan M, Hopkins A, Jaffee EM. Tumor Mutational Burden and Response Rate to PD-1 Inhibition. N Engl J Med 2017;377:2500-1.

22. Briggs SE, Gozzard P, Talbot DC. The association between Lambert-Eaton myasthenic syndrome and small cell lung carcinoma. Immuno Targets Ther 2013;2:31-7.

23. Graus F, Dalmou J, Reñé R, et al. Anti-Hu antibodies in patients with small-cell lung cancer: association with complete response to therapy and improved survival. J Clin Oncol 1997;15:2866-72.

24. Iams WT, Shiuan E, Meador CB, et al. Improved Prognosis and Increased Tumor-Infiltrating Lymphocytes in Patients Who Have SCLC With Neurologic Paraneoplastic Syndromes. J Thorac Oncol 2019;14:1970-81.

25. Arriola E, Wheater M, Galea I, et al. Outcome and
Biomarker Analysis from a Multicenter Phase 2 Study of Ipilimumab in Combination with Carboplatin and Etoposide as First-Line Therapy for Extensive-Stage SCLC. J Thorac Oncol 2016;11:1511-21.

26. Hardy-Werbin M, Arpí O, Taus A, et al. Assessment of neuronal autoantibodies in patients with small cell lung cancer treated with chemotherapy with or without ipilimumab. Oncoimmunology 2017;7:e1395125.

27. Yu H, Batenchuk C, Badzio A, et al. PD-L1 Expression by Two Complementary Diagnostic Assays and mRNA In Situ Hybridization in Small Cell Lung Cancer. J Thorac Oncol 2017;12:110-20.

28. Chung HC, Lopez-Martin JA, Kao SC-H, et al. Phase 2 study of pembrolizumab in advanced small-cell lung cancer (SCLC): KEYNOTE-158. J Clin Oncol 2018;36:8506.

29. Sun Y, Zhai C, Chen X, et al. Characterization of PDL1 protein expression and CD8 + tumor-infiltrating lymphocyte density, and their associations with clinical outcome in small-cell lung cancer. Transl Lung Cancer Res 2019;8:748-59.

30. Doyle A, Martin WJ, Funa K, et al. Markedly decreased expression of class I histocompatibility antigens, protein, and mRNA in human small-cell lung cancer. J Exp Med 1985;161:1135-51.

31. Bonanno L, Pavan A, Dieci MV, et al. The role of immune microenvironment in small-cell lung cancer: Distribution of PD-L1 expression and prognostic role of FOXP3positive tumour infiltrating lymphocytes. Eur J Cancer 2018;101:191-200.

32. FDA grants nivolumab accelerated approval for third-line treatment of metastatic small cell lung cancer. FDA. 2019 Sep 2 [cited 2020 Mar 8]. Available online: http://www. fda.gov/drugs/resources-information-approved-drugs/ fda-grants-nivolumab-accelerated-approval-third-linetreatment-metastatic-small-cell-lung-cancer

33. FDA approves pembrolizumab for metastatic small cell lung cancer. FDA [Internet]. 2019 Dec 20 [cited 2020 Mar 8]. Available online: http://www.fda.gov/drugs/ resources-information-approved-drugs/fda-approvespembrolizumab-metastatic-small-cell-lung-cancer

34. Ready NE, Ott PA, Hellmann MD, et al. Nivolumab Monotherapy and Nivolumab Plus Ipilimumab in Recurrent Small Cell Lung Cancer: Results From the CheckMate 032 Randomized Cohort. J Thorac Oncol 2020;15:426-35.

35. Ready N, Farago AF, de Braud F, et al. Third-Line Nivolumab Monotherapy in Recurrent SCLC: CheckMate 032. J Thorac Oncol 2019;14:237-44. 
36. Ott PA, Elez E, Hiret S, et al. Pembrolizumab in Patients With Extensive-Stage Small-Cell Lung Cancer: Results From the Phase Ib KEYNOTE-028 Study. J Clin Oncol 2017;35:3823-9.

37. Chung HC, Piha-Paul SA, Lopez-Martin J, et al. Pembrolizumab After Two or More Lines of Previous Therapy in Patients With Recurrent or Metastatic SCLC: Results From the KEYNOTE-028 and KEYNOTE-158 Studies. J Thorac Oncol 2020;15:618-27.

38. ESMO. Second-Line Nivolumab Is Not Superior to Chemotherapy in SCLC. [cited 2020 Feb 15]. Available online: https://www.esmo.org/oncology-news/SecondLine-Nivolumab-Is-Not-Superior-to-Chemotherapyin-SCLC

39. Sequist LV, Chiang A, Gilbert J, et al. Clinical activity, safety and predictive biomarkers results from a phase Ia atezolizumab (atezo) trial in extensive-stage small cell lung cancer (ES-SCLC). Ann Oncol 2016;27:vi493-6.

40. Goldman JW, Dowlati A, Antonia SJ, et al. Safety and antitumor activity of durvalumab monotherapy in patients with pretreated extensive disease small-cell lung cancer (ED-SCLC). J Clin Oncol 2018;36:8518.

41. Bondarenko I, Juan-Vidal O, Pajkos G, et al. 1665PDPreliminary efficacy of durvalumab plus tremelimumab in platinum-refractory/resistant ED-SCLC from arm A of the phase II BALTIC study. Ann Oncol 2018;29:iii596-602.

42. Reck M, Luft A, Szczesna A, et al. Phase III Randomized Trial of Ipilimumab Plus Etoposide and Platinum Versus Placebo Plus Etoposide and Platinum in Extensive-Stage Small-Cell Lung Cancer. J Clin Oncol 2016;34:3740-8.

43. Horn L, Mansfield AS, Szczęsna A, et al. First-Line Atezolizumab plus Chemotherapy in Extensive-Stage Small-Cell Lung Cancer. N Engl J Med 2018;379:2220-9.

44. Reck M, Liu SV, Mansfield AS, et al. 1736 O IMpower133: Updated overall survival (OS) analysis of first-line (1L) atezolizumab (atezo) + carboplatin + etoposide in extensive-stage SCLC (ES-SCLC). Ann Oncol 2019;30:v710-1.

45. Liu S. IMpower133: Characterisation of long-term survivors treated first-line with chemotherapy \pm atezolizumab in extensive-stage small cell lung cancer. Ann Oncol 2020;31:S974-87.

46. Mansfield AS, Każarnowicz A, Karaseva N, et al. Safety and patient-reported outcomes of atezolizumab, carboplatin, and etoposide in extensive-stage small-cell lung cancer (IMpower133): a randomized phase I/III trial. Ann Oncol 2020;31:310-7.
47. FDA approves atezolizumab for extensive-stage small cell lung cancer. FDA. 2019 Dec 20 [cited 2020 Mar 30]. Available online: http://www.fda.gov/drugs/drugapprovals-and-databases/fda-approves-atezolizumabextensive-stage-small-cell-lung-cancer

48. CHMP recommends EU approval of Roche's Tecentriq in combination with chemotherapy as an initial treatment of adults with extensive-stage small cell lung cancer. [cited 2020 Mar 30]. Available online: https://www.roche.com/ media/releases/med-cor-2019-07-26.htm

49. Paz-Ares L, Dvorkin M, Chen Y, et al. Durvalumab plus platinum-etoposide versus platinum-etoposide in firstline treatment of extensive-stage small-cell lung cancer (CASPIAN): a randomised, controlled, open-label, phase 3 trial. Lancet 2019;394:1929-39.

50. Paz-Ares LG, Dvorkin M, Chen Y, et al. Durvalumab \pm tremelimumab + platinum-etoposide in first-line extensivestage SCLC (ES-SCLC): Updated results from the phase III CASPIAN study. J Clin Oncol 2020;38:9002.

51. Chen Y, Paz-Ares LG, Dvorkin M, et al. First-line durvalumab plus platinum-etoposide in extensive-stage (ES)-SCLC (CASPIAN): Impact of brain metastases on treatment patterns and outcomes. J Clin Oncol 2020;38:9068.

52. Goldman JW, Garassino MC, Chen Y, et al. Patientreported outcomes with first-line durvalumab plus platinum-etoposide versus platinum-etoposide in extensive-stage small-cell lung cancer (CASPIAN): a randomized, controlled, open-label, phase III study. Lung Cancer 2020;149:46-52.

53. FDA approves durvalumab for extensive-stage small cell lung cancer. FDA. 2020 Mar 30 [cited 2020 Apr 1]. Available online: http://www.fda.gov/drugs/resourcesinformation-approved-drugs/fda-approves-durvalumabextensive-stage-small-cell-lung-cancer

54. Imfinzi confirmed a sustained overall survival benefit in final analysis of the Phase III CASPIAN trial in 1st-line extensive-stage small cell lung cancer. [cited 2020 Mar 29]. Available online: https://www.astrazeneca.com/mediacentre/press-releases/2020/imfinzi-confirmed-a-sustainedoverall-survival-benefit-in-final-analysis-of-the-phase-iiicaspian-trial-in-1st-line-extensive-stage-small-cell-lungcancer.html

55. Long-term ES-SCLC outcomes on durvalumabchemotherapy predicted by PFS duration. medwirenews. com. 2020 [cited 2020 Oct 7]. Available online: https:// www.medwirenews.com/oncology/lung-cancer/caspian-essclc-durvalumab/18415532 
56. Rudin CM, Awad MM, Navarro A, et al. Pembrolizumab or Placebo Plus Etoposide and Platinum as First-Line Therapy for Extensive-Stage Small-Cell Lung Cancer: Randomized, Double-Blind, Phase III KEYNOTE-604 Study. J Clin Oncol 2020;38:2369-79.

57. Martini G, Bria E, Gridelli C, et al. 1808TiP Atezolizumab (ATZ) in combination with carboplatin $(\mathrm{Cb})$ and etoposide (Eto) in the treatment of patients with previously untreated extensive-stage small cell lung cancer (ES-SCLC): A multicenter, phase IIIb, single arm, safety study (MAURIS trial). Ann Oncol 2020;31:S1044-5.

58. New Data Gives Hope to Small Cell Lung Cancer Patients. EORTC. 2020 [cited 2020 Oct 5]. Available online: https://www.eortc.org/blog/2020/09/18/new-datagives-hope-to-small-cell-lung-cancer-patients/

59. Gadgeel SM, Pennell NA, Fidler MJ, et al. Phase II Study of Maintenance Pembrolizumab in Patients with Extensive-Stage Small Cell Lung Cancer (SCLC). J Thorac Oncol 2018;13:1393-9.

60. Owonikoko TK, Kim HR, Govindan R, et al. LBA1_ PRNivolumab (nivo) plus ipilimumab (ipi), nivo, or placebo (pbo) as maintenance therapy in patients (pts) with extensive disease small cell lung cancer (ED-SCLC) after first-line (1L) platinum-based chemotherapy (chemo): Results from the double-blind, randomized phase III CheckMate 451 study. Ann Oncol 2019;30:ii77-80.

61. Thomas M, Ponce-Aix S, Navarro A, et al.

Immunotherapeutic maintenance treatment with toll-like receptor 9 agonist lefitolimod in patients with extensivestage small-cell lung cancer: results from the exploratory, controlled, randomized, international phase II IMPULSE study. Ann Oncol 2018;29:2076-84.

62. Theelen WSME, Peulen HMU, Lalezari F, et al. Effect of Pembrolizumab After Stereotactic Body Radiotherapy vs Pembrolizumab Alone on Tumor Response in Patients With Advanced Non-Small Cell Lung Cancer: Results of the PEMBRO-RT Phase 2 Randomized Clinical Trial. JAMA Oncol 2019;5:1276-82.

63. Perez BA, Kim S, Dilling TJ, et al. A Prospective Single Arm Phase I/II Study: Consolidative Ipilimumab and Nivolumab with Thoracic Radiotherapy after Platinum Based Chemotherapy for Patients with Extensive-Stage Small Cell Lung Cancer. Int J Radiat Oncol Biol Phys 2019;105:S36.

64. Welsh JW, Heymach JV, Chen D, et al. Phase I Trial of Pembrolizumab and Radiation Therapy after Induction Chemotherapy for Extensive-Stage Small Cell Lung Cancer. J Thorac Oncol 2020;15:266-73.
65. Consolidation nivolumab-ipilimumab fails to boost limited-stage SCLC survival after CRT. oncology. medicinematters.com. 2020 [cited 2020 Oct 5]. Available online: https://oncology.medicinematters.com/esmo-2020/ lung-and-thoracic-tumors/stimuli-nivolumab-ipilimumabsclc/18397542

66. Dammeijer F, Lievense LA, Veerman GDM, et al. Efficacy of Tumor Vaccines and Cellular Immunotherapies in NonSmall-Cell Lung Cancer: A Systematic Review and MetaAnalysis. J Clin Oncol 2016;34:3204-12.

67. Giaccone G, Debruyne C, Felip E, et al. Phase III study of adjuvant vaccination with Bec2/bacille CalmetteGuerin in responding patients with limited-disease smallcell lung cancer (European Organisation for Research and Treatment of Cancer 08971-08971B; Silva Study). J Clin Oncol 2005;23:6854-64.

68. Terazaki Y, Yoshiyama K, Matsueda S, et al. Immunological evaluation of personalized peptide vaccination in refractory small cell lung cancer. Cancer Sci 2012;103:638-44.

69. Sakamoto S, Yamada T, Terazaki Y, et al. Feasibility Study of Personalized Peptide Vaccination for Advanced Small Cell Lung Cancer. Clin Lung Cancer 2017;18:e385-94.

70. Krug LM, Ragupathi G, Hood C, et al. Vaccination of patients with small-cell lung cancer with synthetic fucosyl GM-1 conjugated to keyhole limpet hemocyanin. Clin Cancer Res 2004;10:6094-100.

71. Krug LM, Ragupathi G, Hood C, et al. Immunization with $\mathrm{N}$-propionyl polysialic acid-KLH conjugate in patients with small cell lung cancer is safe and induces IgM antibodies reactive with SCLC cells and bactericidal against group B meningococci. Cancer Immunol Immunother CII 2012;61:9-18.

72. Antonia SJ, Mirza N, Fricke I, et al. Combination of p53 cancer vaccine with chemotherapy in patients with extensive stage small cell lung cancer. Clin Cancer Res 2006;12:878-87.

73. Ruotsalainen TM, Mattson K. Review: Interferon Trials in Small Cell Lung Cancer at One Institution: A Comparison of Results Obtained Before and After Initiation of Systematic Treatment Trials Using IFN- $\alpha$ in Combination with Other Modalities. J Interferon Cytokine Res 2002;22:165-71.

74. Balkwill FR, Smyth JF. Interferons in cancer therapy: a reappraisal. Lancet 1987;2:317-9.

75. Ruotsalainen TM, Halme M, Tamminen K, et al. Concomitant chemotherapy and IFN-alpha for small cell lung cancer: a randomized multicenter phase III study. J Interferon Cytokine Res 1999;19:253-9. 
76. Wadler S, Schwartz EL. Antineoplastic Activity of the Combination of Interferon and Cytotoxic Agents against Experimental and Human Malignancies: A Review. Cancer Res 1990;50:3473-86.

77. Zarogoulidis K, Ziogas E, Boutsikou E, et al. Immunomodifiers in combination with conventional chemotherapy in small cell lung cancer: a phase II, randomized study. Drug Des Devel Ther 2013;7:611-7.

78. Ponath P, Menezes D, Pan C, et al. A Novel, Fully Human Anti-fucosyl-GM1 Antibody Demonstrates Potent In Vitro and In Vivo Antitumor Activity in Preclinical Models of Small Cell Lung Cancer. Clin Cancer Res 2018;24:5178-89.

79. Weiskopf K, Jahchan NS, Schnorr PJ, et al. CD47blocking immunotherapies stimulate macrophagemediated destruction of small-cell lung cancer. J Clin Invest 2016;126:2610-20.

80. Hellmann MD, Callahan MK, Awad MM, et al. Tumor Mutational Burden and Efficacy of Nivolumab Monotherapy and in Combination with Ipilimumab in Small-Cell Lung Cancer. Cancer Cell 2018;33:853-861.e4.

81. Kulangara K, Zhang N, Corigliano E, et al. Clinical Utility of the Combined Positive Score for Programmed Death Ligand-1 Expression and the Approval of Pembrolizumab for Treatment of Gastric Cancer. Arch Pathol Lab Med 2019;143:330-7.

82. Fancello L, Gandini S, Pelicci PG, et al. Tumor mutational burden quantification from targeted gene panels: major advancements and challenges. J Immunother Cancer 2019;7:183.

83. Mankor JM, Paats MS, Groenendijk FH, et al. Impact of panel design and cut-off on tumour mutational burden assessment in metastatic solid tumour samples. Br J Cancer 2020;122:953-6.

84. Ott PA, Bang YJ, Piha-Paul SA, et al. T-Cell-Inflamed Gene-Expression Profile, Programmed Death Ligand 1 Expression, and Tumor Mutational Burden Predict Efficacy in Patients Treated With Pembrolizumab Across 20 Cancers: KEYNOTE-028. J Clin Oncol 2019;37:318-27.

85. Marabelle A, Fakih MG, Lopez J, et al. Association of tumour mutational burden with outcomes in patients with select advanced solid tumours treated with pembrolizumab in KEYNOTE-158. Ann Oncol 2019;30:v477-8.

86. Wang Z, Duan J, Cai S, et al. Assessment of Blood Tumor Mutational Burden as a Potential Biomarker for Immunotherapy in Patients With Non-Small Cell Lung Cancer With Use of a Next-Generation Sequencing
Cancer Gene Panel. JAMA Oncol 2019;5:696-702.

87. Schalper K, Carvajal-Hausdorf D. Objective measurement and significance of PD-L1, B7-H3, B7-H4 and TILs in small cell lung cancer (SCLC). J Clin Oncol 2016;34:abstract 8566.

88. Busch SE, Hanke ML, Kargl J, et al. Lung Cancer Subtypes Generate Unique Immune Responses. J Immunol 2016;197:4493-503.

89. He Y, Rozeboom L, Rivard CJ, et al. MHC class II expression in lung cancer. Lung Cancer 2017;112:75-80.

90. Horrigan SK. Replication Study: The CD47-signal regulatory protein alpha $(\mathrm{SIRPa})$ interaction is a therapeutic target for human solid tumors. eLife 2017;6:e18173.

91. Rivalland G, Walkiewicz M, Wright GM, et al. Small cell lung cancer: The immune microenvironment and prognostic impact of checkpoint expression. J Clin Oncol 2017;35:8569.

92. Ricciuti B, Naqash AR, Henick B, et al. OA03.07 Immune-Related Adverse Events and Clinical Outcome to Anti PD-1 Axis Inhibition in SCLC: A Multicenter Retrospective Analysis. J Thorac Oncol 2019;14:S213-4.

93. Murciano-Goroff YR, Hui AB, Chabon JJ, et al. Early ctDNA response assessment for prediction of platinum sensitivity in small cell lung cancer. J Clin Oncol 2020;38:9067.

94. Mohan S, Foy V, Ayub M, et al. Profiling of Circulating Free DNA Using Targeted and Genome-wide Sequencing in Patients with SCLC. J Thorac Oncol 2020;15:216-30.

95. Moding EJ, Liu Y, Nabet BY, et al. Circulating tumor DNA dynamics predict benefit from consolidation immunotherapy in locally advanced non-small-cell lung cancer. Nat Cancer 2020;1:176-83.

96. Almodovar K, Iams WT, Meador CB, et al. Longitudinal cell-free DNA analysis in patients with small cell lung cancer reveals dynamic insights into treatment efficacy and disease relapse. J Thorac Oncol 2018;13:112-23.

97. Wang $\mathrm{Y}$, Deng $\mathrm{W}$, Li N, et al. Combining Immunotherapy and Radiotherapy for Cancer Treatment: Current Challenges and Future Directions. Front Pharmacol 2018;9:185.

98. Slotman BJ, van Tinteren H, Praag JO, et al. Use of thoracic radiotherapy for extensive stage small-cell lung cancer: a phase 3 randomised controlled trial. Lancet 2015;385:36-42.

99. Rudin CM, Poirier JT, Byers LA, et al. Molecular subtypes of small cell lung cancer: a synthesis of human and mouse model data. Nat Rev Cancer 2019;19:289-97. 
100.Hendriks LEL, Menis J, Reck M. Prospects of targeted and immune therapies in SCLC. Expert Rev Anticancer Ther 2019;19:151-67.

101. Sen T, Rodriguez BL, Chen L, et al. Targeting DNA Damage Response Promotes Antitumor Immunity through STING-Mediated T-cell Activation in Small Cell Lung Cancer. Cancer Discov 2019;9:646-61.

102. Thomas A, Vilimas R, Trindade C, et al. Durvalumab in Combination with Olaparib in Patients with Relapsed SCLC: Results from a Phase II Study. J Thorac Oncol 2019;14:1447-57.

103. Goel S, DeCristo MJ, Watt AC, et al. CDK4/6 inhibition triggers anti-tumour immunity. Nature 2017;548:471-5.

104. Forster M, Moreno V, Calvo E, et al. P1.12-20 Overall Survival with Lurbinectedin Plus Doxorubicin in Relapsed SCLC. Results from an Expansion Cohort of a Phase Ib Trial. J Thorac Oncol 2018;13:S581.

105.Paz-Ares LG, Perez JMT, Besse B, et al. Efficacy and safety profile of lurbinectedin in second-line SCLC patients: Results from a phase II single-agent trial. J Clin Oncol 2019;37:8506.

106. Calvo E, Moreno V, Flynn M, et al. Antitumor activity of lurbinectedin (PM01183) and doxorubicin in relapsed small-cell lung cancer: results from a phase I study. Ann Oncol 2017;28:2559-66.

107.Xie W, Forveille S, Iribarren K, et al. Lurbinectedin synergizes with immune checkpoint blockade to generate anticancer immunity. OncoImmunology 2019;8:e1656502.

108. Belgiovine C, Bello E, Liguori M, et al. Lurbinectedin reduces tumour-associated macrophages and the inflammatory tumour microenvironment in preclinical models. Br J Cancer 2017;117:628-38.

109. Owonikoko TK, Dwivedi B, Chen Z, et al. YAP1 positive small-cell lung cancer subtype is associated with the T-cell inflamed gene expression profile and confers good prognosis and long term survival. J Clin Oncol

Cite this article as: Dumoulin DW, Dingemans AMC, Aerts JGJV, Remon J, De Ruysscher DKM, Hendriks LEL. Immunotherapy in small cell lung cancer: one step at a time: a narra-tive review. Transl Lung Cancer Res 2021;10(6):29702987. doi: $10.21037 /$ tlcr-20-630
2020;38:9019.

110. Morgensztern D, Besse B, Greillier L, et al. Efficacy and Safety of Rovalpituzumab Tesirine in ThirdLine and Beyond Patients with DLL3-Expressing, Relapsed/Refractory Small-Cell Lung Cancer: Results From the Phase II TRINITY Study. Clin Cancer Res [Internet]. 2019 Sep 10 [cited 2020 Apr 14]; Available from: https://clincancerres.aacrjournals.org/content/ early/2019/10/12/1078-0432.CCR-19-1133

111. Malhotra J, Nikolinakos P, Leal T, et al. Ph1/2 study of Rova-T in combination with nivolumab (Nivo) \pm ipilimumab (Ipi) for patients (pts) with $2 \mathrm{~L}+$ extensive-stage (ED) SCLC. J Clin Oncol 2019;37:8516.

112.Poirier JT, Dobromilskaya I, Moriarty WF, et al. Selective tropism of Seneca Valley virus for variant subtype small cell lung cancer. J Natl Cancer Inst 2013;105:1059-65.

113.Mollaoglu G, Guthrie MR, Böhm S, et al. MYC Drives Progression of Small Cell Lung Cancer to a Variant Neuroendocrine Subtype with Vulnerability to Aurora Kinase Inhibition. Cancer Cell 2017;31:270-85.

114.Huang Y-H, Klingbeil O, He XY, et al. POU2F3 is a master regulator of a tuft cell-like variant of small cell lung cancer. Genes Dev 2018;32:915-28.

115. Takahashi T, Yamanaka T, Seto T, et al. Prophylactic cranial irradiation versus observation in patients with extensive-disease small-cell lung cancer: a multicentre, randomised, open-label, phase 3 trial. Lancet Oncol 2017;18:663-71.

116.Lukas RV, Gandhi M, O'Hear C, et al. Safety and efficacy analyses of atezolizumab in advanced non-small cell lung cancer (NSCLC) patients with or without baseline brain metastases. Ann Oncol 2017;28:ii28.

117. Berghoff AS, Venur VA, Preusser M, et al. Immune Checkpoint Inhibitors in Brain Metastases: From Biology to Treatment. Am Soc Clin Oncol Educ Book 2016;(36):e116-22. 